\title{
Linear Stability Analysis of Steady State Tropical Cyclones with Single or Double Eyewalls
}

\author{
Ellen K. Luckins and Robert A. Van Gorder* \\ Mathematical Institute, University of Oxford, Andrew Wiles Building, Radcliffe Observatory Quarter, Woodstock Road, Oxford, OX2 \\ 6GG, United Kingdom \\ *Correspondence to: Robert.VanGorder@maths.ox.ac.uk
}

\begin{abstract}
The structure of an eyewall within an intense tropical cyclone is known to strongly influence its intensity. Furthermore, intense tropical cyclones often undergo an eyewall replacement cycle, a process in which an outer eyewall forms, contracts, and replaces an inner eyewall. During this process, there will exist two concentric eyewalls. To examine the effects of such structures, analytical solutions of the transverse circulation equation associated with a balanced vortex model have been proposed in the literature. For a single eyewall, the analytical solution is defined over three regions, the eye, eyewall, and far-field, while in the case of concentric or double eyewalls, one subdivides the domain into five regions, the eye, inner eyewall, moat, outer eyewall, and far-field. The goal of the present paper is to present a stability analysis for steady state vortex solutions corresponding to tropical cyclones with either single or double eyewalls. We first obtain general steady state solutions and a general approach for the stability or instability of such states involving a perturbation analysis (which makes use of small perturbations to the vortex structure in each of the three or five regions). We then make several simplifying assumptions on the form of the stationary solutions so as to make the stability analysis tractable while maintaining physical relevance. The obtained stability criteria depend on the model parameters as well as on the structure of the perturbations the steady state solutions undergo. Making use of parameter values calibrated from the literature, we then provide bifurcation diagrams giving regions of stability or instability. Such analytical stability results for steady state vortex solutions of the transverse circulation equation complement existing numerical stability results in the literature.
\end{abstract}

Key Words: hurricane; eyewall replacement cycle; stability analysis; analytical solution; vortex dynamics

Received...

1. Introduction

The structure of a hurricane or tropical cyclone core generally consists of a single, approximately circular ring of high tangential wind speeds, convection, and storm clouds (the eyewall) surrounding an area of clearer, calmer fluid (the eye). However, under certain circumstances a secondary eyewall (SE) may form around the original (primary or inner) eyewall, giving the hurricane a concentric eyewall (CE) structure. The outer eyewall may then contract, causing the inner eyewall to break down so that the outer eyewall takes over as the only eyewall. The dynamics of this process, called an eyewall replacement cycle (ERC), are still not well understood, although there have been many studies (numerical, analytical and observational) into the mechanisms which may drive various aspects of the process. As a hurricane undergoes an ERC, the intensity of the storm changes greatly, and as such there is a real need to predict when and how a hurricane may form a SE and undergo an ERC in order to forecast this phenomenon well.
There have been many studies into the causes and mechanisms driving SE formation. The work of Nong and Emanuel (2003), for instance, demonstrates the importance of the WISHE mechanism proposed in Emanuel (1987) in amplifying small perturbations outside the primary eyewall (which they hypothesise may be due to some external forcing) into a full concentric eyewall structure. Amongst others, Montgomery and Kallenbach (1997) considered the effect of the interaction between the core vortex and vortex Rossby waves, which was shown to alter the intensity, and accelerate the tangential winds, and so effect the formation of a SE. Dritschel and Waugh (1992), and papers by Kuo et al. (Kuo et al. $(2004,2008)$ ) each describe the formation of a SE from two distinct areas of vorticity. In particular, Kuo et al. (2008) is an idealised numerical study (building on the work of Rozoff et al. (2006)) which examines the effect that different models for the vortices has on the structure of the concentric eyewall setup produced. The effect of ice crystals on the formation and physical properties of a SE are investigated in Zhou and Wang (2011). 
Terwey and Montgomery (2008) use two high-resolution, fullphysics models to analyse a variety of these different hypotheses for the formation of SEs, and includes a summary of these hypotheses and their findings as to each hypothesis' plausibility. This paper also introduces a new mechanism for SE formation which is shown to be supported by the data produced in their models. This new hypothesis (the Beta-Skirt-Axisymmetrisation hypothesis) emphasises the need for an initial beta-skirt surrounding the central vortex which overlaps an area of strong convective potential. The resulting cumulus convection behaves as a source of eddy vorticity generating low-level localised cyclonic jets in the beta-skirt. These can then amplify to redouble convection through from the boundary layer. The importance of the boundary layer is also observed in the mechanism for SE formation proposed by Huang et al. (2012). The boundary layer dynamics of tropical cyclones has recently been investigated more generally in the numerical models of Williams Jr (2016); Williams et al. (2013).

There have also been many studies into the dynamics and evolution of hurricanes with an established CE structure. Yang et al. (2013) developed a classification system for hurricanes with CEs, showing that, although some SEs quickly dissipate without damaging the inner eyewall, and some CE set-ups appear stable for a significant time-period, from the data studied the majority of hurricanes with CE structure undergo an ERC with the outer eyewall contracting while the inner collapses, so that the outer eyewall becomes the sole eyewall of the storm. As well as summarising hypotheses for SE formation, the potential mechanisms driving the ERC are also discussed in Zhou and Wang (2011). The diabatic heating in the outer eyewall is found to cause subsidence in the core in both the analytical study of Rozoff et al. (2008) and the numerical model of Wu et al. (2016) but using the Weather Research and Forecasting (WRF) model, Zhou and Wang show the difficulty in identifying whether the outer eyewall directly causes the break down of the inner through increased subsidence. During the breakdown of the inner eyewall as well as the formation of the outer, there are hypotheses suggesting the boundary layer plays a key role. The effect of the boundary layer may either be direct, with the outer eyewall cutting off boundary layer inflow to the inner eyewall and so reducing the convection there, as in Willoughby et al. (1982), or indirect, with downdrafts in the moat (induced by the SE) choking off convection and vorticity in the inner eyewall by transporting low entropy air down to the boundary layer, as in the work of Shapiro and Willoughby (1982). Zhou and Wang (2011) concludes that the indirect mechanism is non-essential to the breakdown of the inner eyewall, and that the effect of the direct mechanism is dependent on the moat width between the eyewalls.

The intensity changes of a storm as it undergoes an ERC are described in, for example, Kossin (2015) or Zhou and Wang (2011). Zhou and Wang (2011) describe how as the inner eyewall weakens, the storm intensity diminishes, as the hurricane loses its ability to maintain a warm core. The re-intensification occurs as the outer eyewall - the new primary eyewall - strengthens and contracts, rebuilding the warm core. The significance of these intensity changes clearly motivate a better understanding of ERC dynamics, as forecasting the ERC accurately would lead to an improved ability to predict the highly changeable storm strength.

Regarding the stability and instability to vortex structures present in tropical cycles, the three and five region models were explored in Kossin et al. (2000), where unstable solutions wee determined and then, using simulations, the authors were able to evolve them into more stable structures using a nondivergent barotropic model. Empirical support for the application of such models for representing actual hurricane flow fields is found in Kossin and Eastin (2001), Kossin and Schubert
(2001), Kossin et al. (2002), Kossin and Schubert (2003), Kossin and Schubert (2004), and Rozoff et al. (2009). The flow problems were assumed to be nondivergent barotropic with, yet the models still gave results in agreement with observations from real tropical cycles. For instance, the emergence of vortex crystals in Hurricane Isabel (Kossin and Schubert 2004) at least suggests that the underlying assumptions and steady state solutions preserve enough of the physics. Other studies on stability analyses have been undertaken in the analysis of hurricane core structures; for instance, Schubert et al. (1999) examined the linear stability of single eyewalls. The instability they obtain is shown to lead to polygonal-shaped structures. Nolan and Montgomery (2002) consider the linear perturbation dynamics of the development of an axisymmetric vortex. The development of instabilities within the inner core are considered in Naylor and Schecter (2014).

The roles of an expanding wind field and inertial stability in tropical cyclone secondary eyewall formation were considered in Rozoff et al. (2012). Wang et al. (2016) examine secondary eyewall formation (SEF) in an idealized simulation of a tropical cyclone from the perspective of both the balanced and unbalanced dynamics and through the tangential wind (Vt) budget analysis. Abarca and Montgomery (2014) consider departures from axisymmetric balance dynamics during a case of secondary eyewall formation. One main finding of Abarca and Montgomery (2014) is that balance dynamics does not capture the tangential wind spinup during the simulated secondary eyewall formation event. The dynamics of secondary eyewall formation in sheared tropical cyclones was studied in Zhang et al. (2017). Regarding real-world demonstration of theory, Sun et al. (2013) use simulations of Typhoon Sinlaku (2008) to examine the role of peripheral convection in SEF and ERCs.

Motivated by these results, in this paper we look to analyse the linear stability of steady solutions to the modelling equations (as used in Schubert et al. (2007); Rozoff et al. (2008), and justified by the non-dimensionalisation argument in Willoughby (1979)) under various types of perturbations. This is an attempt to analyse the physical situations under which a single or concentric eyewall structure is unstable and perhaps therefore likely to change from one set-up to the other (i.e.: move through phases of an ERC). Two types of solutions are considered, one designed to model a steady state single eyewall hurricane core, and the other a CE structure. To obtain these stability or instability results, we first obtain solutions at steady state. We then introduce a small perturbation to these results, and based on whether this perturbation grows or decays in time, we deduce whether the steady state vortex structures are stable to small perturbations. Mathematically, this will involve solving a linear eigenvalue problem in each of the three or five regions and then matching the solutions. The spectrum of the eigenvalue problems will then inform the choice of stability parameter which determines exponential growth or decay of the perturbations in time.

While stability and instability of such problems has been considered in the Schubert et al. (2007) and Rozoff et al. (2008), note that in obtaining the base solution at lowest order those works made a number of assumptions on the properties of solutions, in order to convert the governing equations into a simpler stream function formulation. Therefore, the solutions they obtain can be viewed as approximations to the lowest order stationary solutions we consider. However, if one directly perturbs their solutions, then the solution to the next order eigenvalue problem, from which stability or instability is ascertained in our approach, will not always give the appropriate spectrum. This would result in a different stability parameter (or perhaps no stability parameter at all) as the linearized first order correction would not be 
linearized around the exact steady state, but rather an approximate steady state. Therefore, while the results and approximations of Schubert et al. (2007) and Rozoff et al. (2008) are certainly of physical interest, their approximate solutions cannot be directly fed into our stability analysis, and hence we construct steady state solutions directly in order to deduce stability or instability of three or five region vortex configurations.

The paper is organised as follows. In Section 2 we start by deriving our own simple, piecewise, barotropic, steady state solutions to the original idealised axisymmetric equations. We set out the general perturbation analysis in Section 3, and a general linear stability analysis of the piecewise steady state solutions is carried out in Section 4. In Section 5 we consider specific perturbations relevant to applications (including perturbations obtained using realistic parameter values) in both the threeregion model (eye, eyewall, and far-field) and five-region model (eye, inner eyewall, moat, outer eyewall, and far-field), and we obtain bifurcation diagrams showing the stability of two different solutions as the perturbation parameters are varied. A brief summary and discussion is provided in Section 6.

\section{Piecewise, continuous, steady state solutions}

As a reminder, when we attempted to use steady state solutions of Schubert et al. (2007) and Rozoff et al. (2008), we found that they did not actually solve the governing equations at lowest order, and hence are not true solutions but rather approximations. While approximate solutions are fine for some contexts, since the governing system is nonlinear, we need to be sure we have exact steady state solutions in order to carry out a meaningful stability analysis. Therefore, we obtain such steady state solutions in this section. As in Schubert et al. (2007) and Rozoff et al. (2008), we shall assume that the tropical cyclone vortex is partitioned into three regions for the single eyewall case, or five regions for the double (concentric) eyewall case.

Using cylindrical log-pressure coordinates, centred on the vortex and moving with it, the fluid velocity $\boldsymbol{u}$ is given by

$$
\boldsymbol{u}=u \boldsymbol{e}_{\boldsymbol{r}}+v \boldsymbol{e}_{\boldsymbol{\theta}}+w \boldsymbol{e}_{\boldsymbol{z}}
$$

where $z=H \log \left(\frac{p_{0}}{p}\right)$ is the $\log$-pressure vertical coordinate and $w=\frac{D z}{D t}$. In this coordinate system and assuming the system is axisymmetric, the equations of motion may be taken (as in Rozoff et al. (2008) and Schubert et al. (2007)) to be:

$$
\begin{gathered}
\left(f+\frac{2 v}{r}\right) \frac{\partial v}{\partial z}=\frac{g}{T_{0}} \frac{\partial T}{\partial r} \\
\frac{\partial v}{\partial t}+u\left(\frac{\partial v}{\partial r}+\frac{v}{r}+f\right)+w \frac{\partial v}{\partial z}=0 \\
\frac{\partial(r u)}{r \partial r}+\frac{\partial w}{\partial z}-\frac{w}{H}=0 \\
\frac{\partial T}{\partial t}+u \frac{\partial T}{\partial r}+w\left(\frac{\partial T}{\partial z}+\frac{R T}{c_{p} H}\right)=\frac{Q}{c_{p}}
\end{gathered}
$$

Equation (4) is precisely the continuity equation (conservation of mass) in these coordinates, and (5) is the thermodynamic equation, where $Q$ is the rate of diabatic heating. Here $f$ is the Coriolis parameter.

The first two equations (2) and (3) come from the three momentum equations, under the assumptions that to leading order the motion is axisymmetric, and that the tangential velocity is of a higher order than the radial and vertical velocities:

$$
u \sim w \sim \epsilon v
$$

for $\epsilon<<1$.
As shown in Willoughby (1979), these assumptions give that the flow is in hydrostatic and gradient balance to leading order, (when combined these equations give the thermal wind equation (2)) and the tangential wind equation (3).

We look for a steady state solution $\hat{v}(r), \hat{u}(r, z), \hat{w}(r, z)$, $\hat{T}(r, z)$. We also assume that to lowest order the velocity $v$ is barotropic (as in Rozoff et al. (2008); Schubert et al. (2007)) so that $\frac{\partial \hat{v}}{\partial z}=0$. Equation (3) therefore reads

$$
\hat{u}\left(\frac{d \hat{v}}{d r}+\frac{\hat{v}}{r}+f\right)=0 .
$$

When $\hat{u} \neq 0$, note that $\hat{v}$ solves $\frac{d \hat{v}}{d r}+\frac{\hat{v}}{r}+f=0$. The solution of this ODE is

$$
\hat{v}(r)=\frac{C}{r}-\frac{f r}{2},
$$

for some constant $C$. Otherwise, if $\hat{u}=0$, then we obtain no information on $\hat{v}$ at lowest order.

We choose in fact, to consider a piecewise solution in $r$, with discontinuities in the solution possible at $r=r_{i}$, where in the three region (single eyewall) model $\left[r_{0}=0, r_{1}\right)$ is the eye, $\left[r_{1}, r_{2}\right)$ is the eyewall, and $\left[r_{2}, r_{3}=\infty\right)$ is the far-field. Similarly, in the five region (concentric eyewall) model, $\left[r_{0}=0, r_{1}\right)$ is the eye, $\left[r_{1}, r_{2}\right.$ ) is the inner eyewall, $\left[r_{2}, r_{3}\right)$ is the moat, $\left[r_{3}, r_{4}\right)$ is the outer eyewall, and $\left[r_{4}, r_{5}=\infty\right)$ is the far-field. Allowing the constants $C$ to be different in each region,

$$
\hat{v}(r)=\hat{v}_{i}(r)=\frac{C_{i}}{r}-\frac{f r}{2}, r \in\left[r_{i}, r_{i+1}\right) .
$$

We also choose the forms of $\hat{w}, Q$ to be

$$
\begin{aligned}
& \hat{w}(r, z)=w_{i}(z), r \in\left[r_{i}, r_{i+1}\right), \\
& Q(r, z)=Q_{i}(z), r \in\left[r_{i}, r_{i+1}\right),
\end{aligned}
$$

where $w_{i}$ and $Q_{i}$ are left arbitrary for the moment.

Equation (4) reads

$$
\frac{\partial(r \hat{u})}{r \partial r}+\frac{\partial \hat{w}}{\partial z}-\frac{\hat{w}}{H}=0
$$

and so taking $\hat{w}=w_{i}(z)$ as above, and solving for $\hat{u}$,

$$
\hat{u}(r, z)=\hat{u}_{i}(r, z)=\frac{U_{i}(z)}{r}+\frac{r}{2}\left(\frac{w_{i}(z)}{H}-w_{i}^{\prime}(z)\right),
$$

for each interval $r \in\left[r_{i}, r_{i+1}\right)$.

The final equation (5) reads

$$
\hat{u} \frac{\partial \hat{T}}{\partial r}+\hat{w}\left(\frac{\partial \hat{T}}{\partial z}+\frac{R \hat{T}}{c_{p} H}\right)=\frac{Q}{c_{p}},
$$

which can be solved generally for $\hat{T}(r, z)=\hat{T}_{i}(z), r \in\left[r_{i}, r_{i+1}\right)$, giving (assuming the functions are integrable)

$$
\begin{aligned}
\hat{T}_{i}(z)=\exp & \left(-\frac{R z}{c_{p} H}\right) \\
\times & \left\{D_{i}+\int^{z} \exp \left(-\frac{R \xi}{c_{p} H}\right) \frac{Q_{i}(\xi)}{c_{p} w_{i}(\xi)} d \xi\right\} .
\end{aligned}
$$

Hence a stationary solution for each sub-interval $r \in\left[r_{i}, r_{i+1}\right)$ may be written as

$\hat{u}(r, z)=\hat{u}_{i}(r, z), \hat{v}(r)=\hat{v}_{i}(r), \hat{w}(r, z)=w_{i}(z), \hat{T}(r, z)=\hat{T}_{i}(z)$

for arbitrary functions $w_{i}(z), Q_{i}(z), U_{i}(z)$ and constants $f_{i}, C_{i}, D_{i}$.

Although we leave most of these arbitrary functions unspecified, in order that the solution is bounded at $r=0$ we 
specify $U_{0}=0$ and $C_{0}=0$. So that the solution is bounded as $r \rightarrow \infty$, we need $w_{2}=f_{2}=0$ for the three region case, and $w_{4}=u_{4}=0$ for the five region case. The condition that $w_{2}=0$ (respectively, $w_{4}=0$ ) means that in the far field, (5) reads $0=\frac{Q_{2}}{c_{p}}$ (respectively, $0=\frac{Q_{4}}{c_{p}}$ ), and so we set $Q_{2}=0$ (respectively, $Q_{4}=$ $0)$. Since the equation for $\hat{T}$ in the far-field region therefore reads $0=0$, there is no information giving the form of $\hat{T}_{2}$ (respectively $\left.\hat{T}_{4}\right)$.

\section{Linear perturbations of the piecewise steady solutions}

Consider a small $\epsilon$-amplitude perturbation of the steady state solutions given by

$$
\begin{gathered}
u(r, z, t)=\hat{u}(r, z)+\epsilon U(r, z, t), \\
v(r, z, t)=\hat{v}(r)+\epsilon V(r, z, t), \\
w(r, z, t)=\hat{w}(r, z)+\epsilon W(r, z, t), \\
T(r, z, t)=\hat{T}(r, z)+\epsilon \tau(r, z, t),
\end{gathered}
$$

so that $U, V, W$, and $\tau$ are order $O(1)$ in the small $\epsilon$ parameter.

Substituting into the equations of motion, we obtain the steady state equations solved by $\hat{u}, \hat{v}, \hat{w}$ and $\hat{T}$ at first order. And at $O(\epsilon)$, considering the local behaviour within a region (so that $\frac{\partial \hat{T}}{\partial r}=0$ ):

$$
\begin{gathered}
\left(f+\frac{2 \hat{v}}{r}\right) \frac{\partial V}{\partial z}=\frac{g}{T_{0}} \frac{\partial \tau}{\partial r} \\
\frac{\partial V}{\partial t}+\hat{u}\left(\frac{\partial V}{\partial r}+\frac{V}{r}\right)+U\left(\frac{d \hat{v}}{d r}+\frac{\hat{v}}{r}+f\right)+\hat{w} \frac{\partial V}{\partial z}=0, \\
\frac{\partial U}{\partial r}+\frac{U}{r}+\frac{\partial W}{\partial z}-\frac{W}{H}=0, \\
\frac{\partial \tau}{\partial t}+\hat{u} \frac{\partial \tau}{\partial r}+\hat{w}\left(\frac{\partial \tau}{\partial z}+\frac{R \tau}{c_{p} H}\right)+W\left(\frac{\partial \hat{T}}{\partial z}+\frac{R \hat{T}}{c_{p} H}\right)=0 .
\end{gathered}
$$

Note that when $\hat{w} \neq 0$ and $\hat{u} \neq 0$, and with $\frac{\partial \hat{T}_{i}}{\partial r}=0$ in each region, we have

$$
\frac{\partial \hat{T}}{\partial z}+\frac{R \hat{T}}{c_{p} H}=\frac{Q}{c_{p} \hat{w}} \quad \text { and } \quad \frac{d \hat{v}}{d r}+\frac{\hat{v}}{r}+f=0,
$$

so these equations for the first order perturbations may be written as

$$
\begin{gathered}
\left(f+\frac{2 \hat{v}}{r}\right) \frac{\partial V}{\partial z}=\frac{g}{T_{0}} \frac{\partial \tau}{\partial r}, \\
\frac{\partial V}{\partial t}+\hat{u}\left(\frac{\partial V}{\partial r}+\frac{V}{r}\right)+\hat{w} \frac{\partial V}{\partial z}=0, \\
\frac{\partial U}{\partial r}+\frac{U}{r}+\frac{\partial W}{\partial z}-\frac{W}{H}=0, \\
\frac{\partial \tau}{\partial t}+\hat{u} \frac{\partial \tau}{\partial r}+\hat{w}\left(\frac{\partial \tau}{\partial z}+\frac{R \tau}{c_{p} H}\right)+\frac{W Q}{c_{p} \hat{w}}=0,
\end{gathered}
$$

in the case where $\hat{u} \neq 0, \hat{w} \neq 0$. We see that (26) is an equation for $V$ only, and so we attempt to solve this first. Then an expression for $\tau$ can be obtained from (25), which in turn gives $W$ through (28) and then $U$ through (27).

Similarly, in the outer or far-field region, to avoid growth in $r$ we shall take $\hat{u}=0$ and $\hat{w}=0$, obtaining

$$
\begin{gathered}
\left(f+\frac{2 \hat{v}}{r}\right) \frac{\partial V}{\partial z}=\frac{g}{T_{0}} \frac{\partial \tau}{\partial r}, \\
\frac{\partial V}{\partial t}+U\left(\frac{d \hat{v}}{d r}+\frac{\hat{v}}{r}+f\right)=0, \\
\frac{\partial U}{\partial r}+\frac{U}{r}+\frac{\partial W}{\partial z}-\frac{W}{H}=0, \\
\frac{\partial \tau}{\partial t}+W\left(\frac{\partial \hat{T}}{\partial z}+\frac{R \hat{T}}{c_{p} H}\right)=0 .
\end{gathered}
$$

Earlier we commented that for $u \hat{=} 0$ and $\hat{w}=0$ we have no information on the functions $\hat{v}$ and $\hat{T}$. We shall therefore determine their form so that the outer solutions match with the neighboring interior solutions.
4. General linear stability of solutions with piecewise constant $U_{i}$ and $w_{i}$

We look for a separable solution of the form

$$
V(r, z, t)=\mathcal{V}_{1}(z) \mathcal{V}_{2}(r, t)
$$

and using this in the above equation this gives

$$
\begin{aligned}
& \mathcal{V}_{1} \frac{\partial \mathcal{V}_{2}}{\partial t}+U_{i}(z) \mathcal{V}_{1} \frac{1}{r}\left(\frac{\partial \mathcal{V}_{2}}{\partial r}+\frac{\mathcal{V}_{2}}{r}\right) \\
& \quad+\left(\frac{w_{i}(z)}{H}-w_{i}^{\prime}(z)\right) \mathcal{V}_{1} \frac{r}{2}\left(\frac{\partial \mathcal{V}_{2}}{\partial r}+\frac{\mathcal{V}_{2}}{r}\right)+w_{i}(z) \frac{d \mathcal{V}_{1}}{d z} \mathcal{V}_{2}=0
\end{aligned}
$$

In order that the $z$-dependence of each term cancels, we require that $\hat{u}_{i}(z)=U_{i}$ and $\hat{w}_{i}(z)=w_{i}$ are constant and that $k_{i} \mathcal{V}_{1}=$ $w_{i} \frac{d \mathcal{V}_{1}}{d z}$. Then, up to a multiplying constant, and in the regions where $w_{i} \neq 0, \mathcal{V}_{1}(z)=e^{\frac{k_{i} z}{w_{i}}}$.

Canceling out the $z$-dependence, for the $w_{i} \neq 0$ case, $\mathcal{V}_{2}$ is governed by the equation

$$
\frac{\partial \mathcal{V}_{2}}{\partial t}+\frac{U_{i}}{r}\left(\frac{\partial \mathcal{V}_{2}}{\partial r}+\frac{\mathcal{V}_{2}}{r}\right)+\frac{w_{i} r}{2 H}\left(\frac{\partial \mathcal{V}_{2}}{\partial r}+\frac{\mathcal{V}_{2}}{r}\right)+k_{i} \mathcal{V}_{2}=0
$$

Setting $\mathcal{V}_{2}(r, t)=\mathcal{V}_{3}(r) \mathcal{V}_{4}(t)$ we see this equation is separable, giving $\mathcal{V}_{4}(t)=e^{\lambda t}$, where $\lambda$ is the separability constant. The spatial equation for $\mathcal{V}_{3}$ reads

$$
\frac{\mathcal{V}_{3}^{\prime}}{\mathcal{V}_{3}}=-2 H r \frac{\lambda+k_{i}}{w_{i} r^{2}+2 H U_{i}}-\frac{1}{r}
$$

and this is valid so long as $w_{i} r^{2}+2 H U_{i} \neq 0$. Integrating (up to a constant), in the regions where $w_{i} \neq 0$ :

$$
\log \left(\mathcal{V}_{3}\right)=\log \left(\frac{\left|w_{i} r^{2}+2 H U_{i}\right|^{-H\left(\lambda+k_{i}\right) / w_{i}}}{r}\right) .
$$

Therefore up to a multiplying constant, we have

$$
\mathcal{V}_{3}(r)=\frac{\left|w_{i} r^{2}+2 H U_{i}\right|^{-H\left(\lambda+k_{i}\right) / w_{i}}}{r} .
$$

For the outer or far-field region, $w_{i}=0$ and $U_{i}=0$, the perturbed equations are instead (29)(32). We need for the solutions to match with solutions in the interior region, hence we assume that they agree in $z$ and $t$ by including the relevant exponential factors $e^{\lambda t}$ and $e^{\mu z}$. We also ensure that the perturbations decay as $r \rightarrow \infty$. Therefore, we consider perturbations of the form

$$
(U, V, W, \tau)=e^{\lambda t} e^{\mu z}(\lambda \mathcal{U}(r), \mathcal{V}(r), \lambda \mathcal{W}(r), \mathcal{T}(r))
$$

within the outer or far-field region. For consistency of this assumption in (29)-(32), we must have that

$$
\frac{\partial \hat{T}}{\partial z}+\frac{R \hat{T}}{c_{p} H}=\hat{T}_{\infty}
$$

where $\hat{T})_{\infty} \neq 0$ is a constant. Solving this, we see that in the farfield the lowest order thermal solution is

$$
\hat{T}=\frac{\hat{T}_{\infty} c_{p} H}{R}+\hat{T}_{\infty}^{\prime} \exp \left(-\frac{R z}{c_{p} H}\right),
$$

where $\hat{T}_{\infty}^{\prime}$ is another constant. This gives a system of equation for the radial components of the perturbation terms in (39):

$$
\mu\left(f+\frac{2 \hat{v}}{r}\right) \mathcal{V}=\frac{g}{T_{0}} \frac{d \mathcal{T}}{d r},
$$




$$
\begin{gathered}
\mathcal{V}+\mathcal{U}\left(\frac{d \hat{v}}{d r}+\frac{\hat{v}}{r}+f\right)=0 \\
\frac{d \mathcal{U}}{d r}+\frac{\mathcal{U}}{r}+\left(\mu-\frac{1}{H}\right) \mathcal{W}=0 \\
\mathcal{T}+\hat{T}_{\infty} \mathcal{W}=0
\end{gathered}
$$

We may isolate $\mathcal{V}, \mathcal{W}, \mathcal{T}$ in terms of $\mathcal{U}$ using (43)-(45),

$$
\begin{gathered}
\mathcal{W}(r)=-\frac{1}{\mu-H^{-1}}\left(\frac{d \mathcal{U}}{d r}+\frac{\mathcal{U}}{r}\right), \\
\mathcal{T}(r)=\frac{\hat{T}_{\infty}}{\mu-H^{-1}}\left(\frac{d \mathcal{U}}{d r}+\frac{\mathcal{U}}{r}\right), \\
\mathcal{V}(r)=-\mathcal{U}(r)\left(\frac{d \hat{v}}{d r}+\frac{\hat{v}}{r}+f\right),
\end{gathered}
$$

and placing these expressions for $\mathcal{V}$ and $\mathcal{T}$ into (42), we obtain and ODE for $\mathcal{U}$. This equation will depend on the form of the perturbation $\hat{v}(r)$, which we recall was arbitrary in the far-field region. Choosing $\hat{v}(r)=\frac{\hat{v}_{\infty}}{r}$ (which exhibits appropriate decay as $r \rightarrow \infty$ ), we have that

$$
\frac{d^{2} \mathcal{U}}{d r^{2}}+\frac{1}{r} \frac{d \mathcal{U}}{d r}-\left(\frac{\chi_{1}}{r^{2}}-\chi_{2}\right) \mathcal{U}=0
$$

Here,

$$
\chi_{1}=1-\frac{2 \mu\left(\mu-H^{-1}\right) T_{0} f \hat{v}_{0}}{\hat{T}_{\infty} g}
$$

and

$$
\chi_{2}=\frac{2 \mu\left(\mu-H^{-1}\right) T_{0} f^{2}}{\hat{T}_{\infty} g} .
$$

Note that $\chi_{1}>0$ for physically relevant parameters (and we can also control this by choosing the arbitrary parameters $\hat{T}_{\infty}$ and $\hat{v}_{\infty}$ ), while the sign of $\chi_{2}$ could be positive or negative.

Solving (49) under the condition $\mathcal{U} \rightarrow 0$ as $r \rightarrow \infty$, we obtain

$$
\mathcal{U}(r)=\tilde{U} \begin{cases}K_{\sqrt{\chi_{1}}}\left(\sqrt{-\chi_{2}} r\right), & \chi_{2}<0 \\ r^{-\sqrt{\chi_{1}}}, & \chi_{2}=0 \\ J_{\sqrt{\chi_{1}}}\left(\sqrt{\chi_{2}} r\right), & \chi_{2}>0\end{cases}
$$

Here $\tilde{U}$ is an arbitrary constant, while $K$ and $J$ are the relevant Bessel functions. Under an appropriate scaling, we may therefore represent the perturbation $V$ as

$$
V(r, z, t)=V_{j} e^{\lambda t} e^{\mu z} \mathcal{V}(r)
$$

where

$$
\mathcal{V}(r)= \begin{cases}K_{\sqrt{\chi_{1}}}\left(\sqrt{-\chi_{2}} r\right), & \chi_{2}<0 \\ r^{-\sqrt{\chi_{1}}}, & \chi_{2}=0 \\ J_{\sqrt{\chi_{1}}}\left(\sqrt{\chi_{2}} r\right), & \chi_{2}>0\end{cases}
$$

Clearly, $V(r, z, t) \rightarrow 0$ as $r \rightarrow \infty$, as needed.

\subsection{Summary of perturbation forms}

The form of the perturbation of the tangential velocity, $V$, in the three region (single eyewall) model is therefore

$$
V(r, z, t)=\left\{\begin{array}{l}
V_{0} \frac{e^{\frac{k_{0} z}{w_{0}}} e^{\lambda t}\left|w_{0} r^{2}\right|^{-H\left(\lambda+k_{0}\right) / w_{0}}}{r}, r \in\left[0, r_{1}\right), \\
V_{1} \frac{e^{\frac{k_{1} z}{w_{1}}} e^{\lambda t}\left|w_{1} r^{2}+2 H U_{1}\right|^{-H\left(\lambda+k_{1}\right) / w_{1}}}{r}, r \in\left[r_{1}, r_{2}\right), \\
V_{2} e^{\mu z} e^{\lambda t} \mathcal{V}(r), r \in\left[r_{2}, \infty\right),
\end{array}\right.
$$

while in the five region (concentric eyewall) model it is

$$
V(r, z, t)=\left\{\begin{array}{l}
V_{0} e^{\frac{k_{0} z}{w_{0}}} e^{\lambda t} \frac{\left|w_{0} r^{2}\right|^{-\frac{H\left(\lambda+k_{0}\right)}{w_{0}}}}{r}, r \in\left[0, r_{1}\right), \\
V_{1} e^{\frac{k_{1} z}{w_{1}}} e^{\lambda t} \frac{\left|w_{1} r^{2}+2 H U_{1}\right|^{-\frac{H\left(\lambda+k_{1}\right)}{w_{1}}}}{r}, r \in\left[r_{1}, r_{2}\right), \\
V_{2} e^{\frac{k_{2} z}{w_{2}}} e^{\lambda t} \frac{\left|w_{2} r^{2}+2 H U_{2}\right|^{-\frac{H\left(\lambda+k_{2}\right)}{w_{2}}}}{r}, r \in\left[r_{2}, r_{3}\right), \\
V_{3} e^{\frac{k_{3} z}{w_{3}}} e^{\lambda t} \frac{\left|w_{3} r^{2}+2 H U_{3}\right|^{-\frac{H\left(\lambda+k_{2}\right)}{w_{3}}}}{r}, r \in\left[r_{3}, r_{4}\right), \\
V_{4} e^{\mu z} e^{\lambda t} \mathcal{V}(r), r \in\left[r_{4}, \infty\right),
\end{array}\right.
$$

for $\mathcal{V}(r)$ defined in (54) and constants $V_{i}$, so long as $U_{2} \neq 0$ in the three region case (respectively $U_{4} \neq 0$ in the five region case).

Note that, as described above, expressions for $\tau$, then $W$, then $U$ can now be calculated from equations (25), (28), and (27), respectively. Since these are linear in $U, V, W$ and $\tau$, each has the same $t$-dependence, namely a factor of $e^{\lambda t}$. The stability of the steady state solution in either of the three or five region cases is therefore the same for each of $\hat{u}, \hat{v}, \hat{w}$, and $\hat{T}$, and is given by the sign of $\lambda$ : if $\lambda>0$ then the solution is unstable, while if $\lambda<0$ then the solution is stable.

In either the three or five region case, we wish to apply the boundary conditions $V=0$ at $r=0$ and as $r \rightarrow \infty$, and also to impose that $V$ is continuous across $r=r_{i}$ for all $z$ and $t$. This fixes $\nu_{i}(z)=e^{\frac{k_{i} z}{w_{i}}}$ for all $z$, so that $\mu:=\frac{k_{i}}{w_{i}}$ is constant for all $i$. This also determines internal matching conditions, which we discuss for the two distinct models separately.

\subsection{Stability in the three region model}

The various boundary and matching conditions require that, at $r=0$ :

$$
\left(\frac{\lambda}{w_{0}}+\mu\right) H<-\frac{1}{2}
$$

at $r=r_{1}$ :

$$
V_{0}\left|w_{0} r_{1}^{2}\right|^{-H\left(\frac{\lambda}{w_{0}}+\mu\right)}=V_{1}\left|w_{1} r_{1}^{2}+2 H U_{1}\right|^{-H\left(\frac{\lambda}{w_{1}}+\mu\right)},
$$

at $r=r_{2}$ :

$$
V_{1}\left|w_{1} r_{2}^{2}+2 H U_{1}\right|^{-H\left(\frac{\lambda}{w_{1}}+\mu\right)}=V_{2} r_{2} \mathcal{V}\left(r_{2}\right),
$$

and as $r \rightarrow \infty$ :

$$
V \rightarrow 0
$$

The $r \rightarrow \infty$ condition is always satisfied due to the choice of $\mathcal{V}(r)$.

We choose to solve (58) for $\lambda$ and then choose the ratio $V_{2} / V_{1}$ so that (59) holds. Taking logs of (58), and rearranging gives

$$
\lambda=\frac{\frac{1}{H} \log \left(\frac{V_{1}}{V_{0}}\right)+\mu \log \left(\frac{\left|w_{0} r_{1}^{2}\right|}{\left|w_{1} r_{1}^{2}+2 H U_{1}\right|}\right)}{\log \left(\left|w_{1} r_{1}^{2}+2 H U_{1}\right|^{1 / w_{1}}\left|w_{0} r_{1}^{2}\right|^{-1 / w_{0}}\right)} .
$$

The sign of $\lambda$ depends on the relative size of the perturbation in the eye and eyewall regions, the $z$-dependence of the perturbation from which we get $\mu$ (since the perturbation $V$ has factor $e^{\mu z}$ ), and the other parameters $r_{i}, w_{i}, U_{i}$ which specify the piecewise steady solution. Hence, the stability or instability of a steady solution will depend strongly on the form of such a solution.

For physically relevant sets of the steady state parameters, we will argue that the denominator of (61) is positive. In the initial derivation of (2)-(5), it was assumed that the magnitudes of the radial and vertical wind speeds are the same order of magnitude, and both smaller than the tangential wind speed,

$$
\frac{U_{i}}{r}+\frac{r w_{i}}{2 H}=\hat{u} \sim \hat{w}=w_{i}
$$


Note that $\left|w_{1} r_{1}^{2}+2 H U_{1}\right|=2 H r_{1}\left|\hat{u}_{1}\left(r_{1}\right)\right|$ and since $U_{0}=0$, we have $\left|w_{0} r_{1}^{2}\right|=2 H r_{1}\left|\hat{u}_{0}\left(r_{1}\right)\right|$. For physically relevant values, following for instance Schubert et al. (2007) or Willoughby (1979), we expect that the radii $r_{i} \sim O\left(10^{4}\right) \mathrm{m}$, and $\hat{u} \sim$ $O(1) \mathrm{ms}^{-1} . H=\frac{R T_{0}}{g}$, the height scale, is $O\left(10^{3}\right) \mathrm{m}$. And so both $\left|w_{1} r_{1}^{2}+2 H U_{1}\right|,\left|w_{0} r_{1}^{2}\right| \sim O\left(10^{7}\right)$. So clearly we expect $\mid w_{1} r_{1}^{2}+$ $2 H U_{1}|,| w_{0} r_{1}^{2} \mid>>1$. We also expect convection in the eyewall, and subsidence in the eye, so that $w_{0}<0$ and $w_{1}>0$, and therefore $\left|w_{1} r_{1}^{2}+2 H U_{1}\right|^{1 / w_{1}}>1$ and $\left|w_{0} r_{1}^{2}\right|^{-1 / w_{0}}>1$ as both powers are positive. Hence, $\log \left(\left|w_{1} r_{1}^{2}+2 H U_{1}\right|^{1 / w_{1}}\left|w_{0} r_{1}^{2}\right|^{-1 / w_{0}}\right)>$ 0 and the denominator of (61) is positive.

The sign of $\lambda$ is therefore the same as the sign of the numerator of (61), and so depends heavily on the form of the perturbation:

$$
\operatorname{sgn}(\lambda)=\operatorname{sgn}\left(\frac{1}{H} \log \left(\frac{V_{1}}{V_{0}}\right)+\mu \log \left(\frac{\left|w_{0} r_{1}^{2}\right|}{\left|w_{1} r_{1}^{2}+2 H U_{1}\right|}\right)\right) .
$$

Consider the ratio

$$
\frac{\left|w_{0} r_{1}^{2}\right|}{\left|w_{1} r_{1}^{2}+2 H U_{1}\right|}=\frac{2 H r_{1}\left|\hat{u}_{0}\left(r_{1}\right)\right|}{2 H r_{1}\left|\hat{u}_{1}\left(r_{1}\right)\right|}=\frac{\left|\hat{u}_{0}\left(r_{1}\right)\right|}{\left|\hat{u}_{1}\left(r_{1}\right)\right|} .
$$

One might expect (or hope) that this ratio is approximately 1 , but recall that no continuity requirement was placed on the piecewise steady radial velocity $\hat{u}$ and so this may well not be the case. In practise, the $w_{i}$ would be chosen to best mimic the azimuthally averaged vertical velocity data in each region, and then the $U_{i}$ would be chosen to ensure that $\hat{u}_{i}=\frac{U_{i}}{r}+\frac{w_{i} r}{2 H}$ best mimics the average radial velocity data. It is highly plausible that in order to best approximate the data, the $U_{i}$ might be chosen so that $\hat{u}$ is not continuous across the boundaries between regions. The sign of $\log \left(\frac{\left|w_{0} r_{1}^{2}\right|}{\left|w_{1} r_{1}^{2}+2 H U_{1}\right|}\right)$ depends on the relative sizes of the $|\hat{u}|$ in the neighbouring regions. Along with details of the perturbation type (specifically the size and sign of the $z$-dependence parameter $\mu$ and the relative sizes of the perturbation in the neighbouring regions $\frac{V_{1}}{V_{0}}$ ), this determines the sign of $\lambda$, and the stability of the steady state solution under this perturbation.

To better visualize what is going on with these perturbations, we give a schematic plot of the perturbation solution (the order zero steady state solution plus the first order correction) in Figure 1. We find that the general form of the perturbation solutions is qualitatively similar to the plots contained in Figure 5 of Schubert et al. (2007) for the tangential and vertical wind velocities, as well as for the temperature profile.

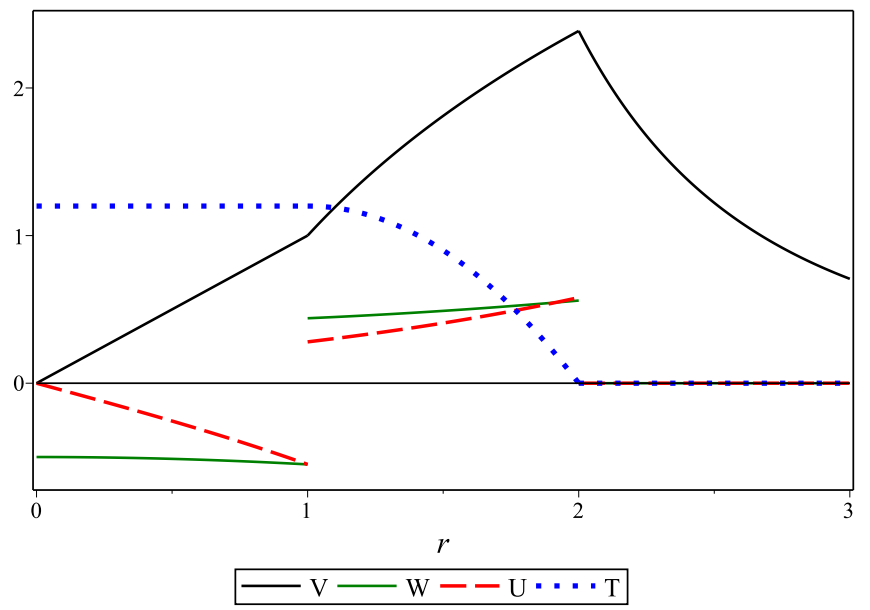

Figure 1. Schematic diagram of the perturbation solutions for non-dimensional $V, W, U$, and $T$. We scale the domain so that $r_{0}=0, r_{1}=1, r_{2}=2$, and fix other parameters at simple values to obtain non-dimensional illustrative plots. This demonstrates that the general form of the perturbations is akin to the three-region results shown in Figure 5 of Schubert et al. (2007) for the tangential and vertical wind, as well as for the temperature profile.

\subsection{Stability in the five region model}

The corresponding boundary and matching conditions in the five region case are, at $r=0$ :

$$
\left(\frac{\lambda}{w_{0}}+\mu\right) H<-\frac{1}{2}
$$

at $r=r_{1}$ :

$$
V_{0}\left|w_{0} r_{1}^{2}\right|^{-H\left(\frac{\lambda}{w_{0}}+\mu\right)}=V_{1}\left|w_{1} r_{1}^{2}+2 H U_{1}\right|^{-H\left(\frac{\lambda}{w_{1}}+\mu\right)},
$$

at $r=r_{2}$ :

$$
V_{1}\left|w_{1} r_{2}^{2}+2 H U_{1}\right|^{-H\left(\frac{\lambda}{w_{1}}+\mu\right)}=V_{2}\left|w_{2} r_{2}^{2}+2 H U_{2}\right|^{-H\left(\frac{\lambda}{w_{2}}+\mu\right)},
$$

at $r=r_{3}$ :

$$
V_{2}\left|w_{2} r_{3}^{2}+2 H U_{2}\right|^{-H\left(\frac{\lambda}{w_{2}}+\mu\right)}=V_{3}\left|w_{3} r_{3}^{2}+2 H U_{3}\right|^{-H\left(\frac{\lambda}{w_{3}}+\mu\right)},
$$

at $r=r_{4}$ :

$$
V_{3}\left|w_{3} r_{4}^{2}+2 H U_{3}\right|^{-H\left(\frac{\lambda}{w_{3}}+\mu\right)}=V_{4} r_{4} \mathcal{V}\left(r_{4}\right),
$$

and as $r \rightarrow \infty$ :

$$
V \rightarrow 0
$$

The $r \rightarrow \infty$ condition is always satisfied due to the choice of $\mathcal{V}(r)$.

In the same way as in the three region case, the other matching conditions (66)-(68) may each be solved individually for $\lambda$ in terms of $V_{i}, \mu$ and the constants describing the steady state solution, namely $w_{i}, r_{i}$, and $U_{i}$, giving three expressions for $\lambda$ (depending on which conditions are used):

$$
\begin{gathered}
\lambda=\frac{\frac{1}{H} \log \left(\frac{V_{1}}{V_{0}}\right)+\mu \log \left(\frac{\left|w_{0} r_{1}^{2}\right|}{\left|w_{1} r_{1}^{2}+2 H U_{1}\right|}\right)}{\log \left(\left|w_{1} r_{1}^{2}+2 H U_{1}\right|^{1 / w_{1}}\left|w_{0} r_{1}^{2}\right|^{-1 / w_{0}}\right)}, \\
\lambda=\frac{\frac{1}{H} \log \left(\frac{V_{2}}{V_{1}}\right)+\mu \log \left(\frac{\left|w_{1} r_{2}^{2}+2 H U_{1}\right|}{\left|w_{2} r_{2}^{2}+2 H U_{2}\right|}\right)}{\log \left(\left|w_{2} r_{2}^{2}+2 H U_{2}\right|^{1 / w_{2}}\left|w_{1} r_{2}^{2}+2 H U_{1}\right|^{-1 / w_{1}}\right)}, \\
\lambda=\frac{\frac{1}{H} \log \left(\frac{V_{3}}{V_{2}}\right)+\mu \log \left(\frac{\left|w_{2} r_{3}^{2}+2 H U_{2}\right|}{\left|w_{3} r_{3}^{2}+2 H U_{3}\right|}\right)}{\log \left(\left|w_{3} r_{3}^{2}+2 H U_{3}\right|^{1 / w_{3}}\left|w_{2} r_{3}^{2}+2 H U_{2}\right|^{-1 / w_{2}}\right)} .
\end{gathered}
$$

For consistency, each of these three expressions must be equal, and the constants $V_{i}$ must be chosen so that they are equal (since we are choosing $V$ to satisfy all the boundary and matching conditions).

As argued in the three region case, the denominator of (71) is positive for physically relevant steady state parameter values, since $w_{0}<0$ and $w_{1}>0$. We expect convection in the secondary eyewall and subsidence in the moat, $w_{2}<0$ and $w_{3}>0$, and so by a similar argument, the denominator of (73) is also always positive for physically relevant data, as both $\left|w_{3} r_{3}^{2}+2 H U_{3}\right|, \mid w_{2} r_{3}^{2}+$ $2 H U_{2} \mid>1$ and the powers $\frac{1}{w_{3}},-\frac{1}{w_{2}}>0$. Conversely, we have $\left|w_{2} r_{2}^{2}+2 H U_{2}\right|,\left|w_{1} r_{2}^{2}+2 H U_{1}\right|>1,-\frac{1}{w_{1}}, \frac{1}{w_{2}}<0$ and so

$$
\left|w_{2} r_{2}^{2}+2 H U_{2}\right|^{1 / w_{2}}\left|w_{1} r_{2}^{2}+2 H U_{1}\right|^{-1 / w_{1}}<1,
$$

hence the denominator of (72) is negative. Therefore, the sign of $\lambda$ must be the same as the sign of the numerators of (71) and (73), which must be the same, and the sign of $\lambda$ must be the opposite of the sign of the numerator of (72). The constants $V_{i}, \mu$ (features of the perturbation) must be chosen such that these conditions are all the same, and, as in the three region case, the sign of $\lambda$ depends on the values of $V_{i}, \mu$ (i.e., the form of the perturbation), as well as the relative sizes of $|\hat{u}|$ across the boundaries between regions. 
The stability criteria obtained for the three and five region steady state configurations are still fairly complicated, although in a way this is desirable as it means that one may insert their own parameter values for their steady state configuration of interest. Still, in order to determine more concrete results, we shall make some physically relevant simplifications in Section 5.

To better visualize what the structure of the perturbations for concentric eyewalls, we give a schematic plot of the perturbation solution (the order zero steady state solution plus the first order correction) in Figure 2. We find that the general form of the perturbation solutions is qualitatively similar to the plots contained in Figure 3 of Rozoff et al. (2008) for the tangential and vertical wind velocities, as well as for the temperature profile.

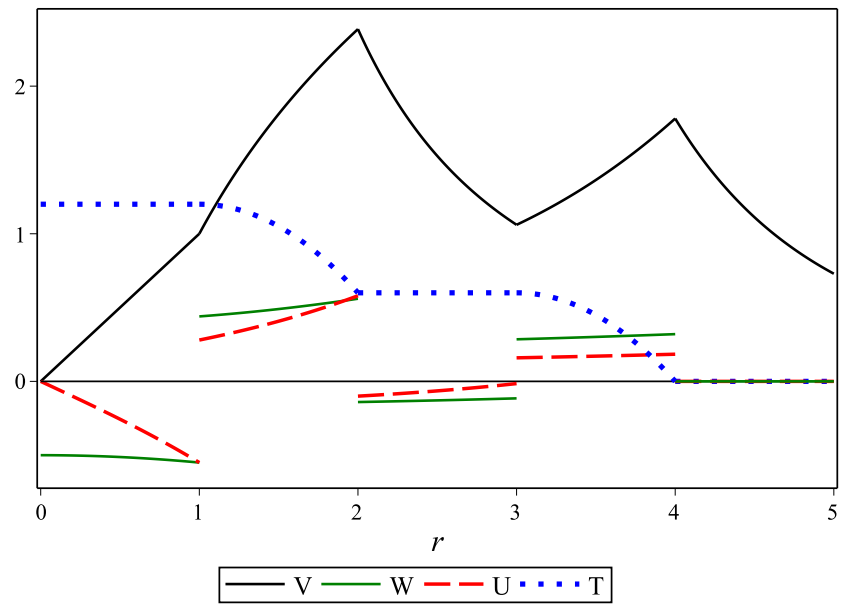

Figure 2. Schematic diagram of the perturbation solutions for non-dimensional $V$, $W, U$, and $T$. We scale the domain so that $r_{0}=0, r_{1}=1, r_{2}=2, r_{3}=3, r_{4}=$ 4 , and fix other parameters at simple values to obtain non-dimensional illustrative plots. This demonstrates that the general form of the perturbations is akin to the five-region results shown in Figure 3 of Rozoff et al. (2008) for the tangential and vertical wind, as well as for the temperature profile.

\subsection{General features of the perturbations}

Before looking at specific perturbations (i.e. specific values of the $V_{i}$ and $\mu$ ), we show some general features of the constants $V_{i}$.

First note that the $V_{i}$ all have the same sign. This is clear from the conditions (58) and (59), (or (66)-(69) in the five region case), since rearranging, $\frac{V_{i}}{V_{i+1}}$ is a ratio of two absolute values, and hence is positive, for all $i$.

Note that the $V_{i}$ satisfy a consistency condition. In the three region case, the conditions (58) and (59) are substituted into (57) to eliminate the $H\left(\frac{\lambda}{w_{i}}+\mu\right)$ factors, giving

$$
\begin{aligned}
-\frac{1}{2}> & \left(\frac{\lambda}{w_{0}}+\mu\right) H \\
& =\frac{\log \left(\frac{V_{0}}{V_{1}}\right)+\left(\frac{\lambda}{w_{1}}+\mu\right) H \log \left(\left|w_{1} r_{1}^{2}+2 H U_{1}\right|\right)}{\log \left(\left|w_{0} r_{1}^{2}\right|\right)} \\
& =\frac{\log \left(\frac{V_{0}}{V_{1}}\right)+\frac{\log \left(\left|w_{1} r_{1}^{2}+2 H U_{1}\right|\right)}{\log \left(\left|w_{1} r_{2}^{2}+2 H U_{1}\right|\right)}\left(\log \left(\frac{V_{1}}{V_{2}}\right)-\log \left(r_{2} \mathcal{V}\left(r_{2}\right)\right)\right)}{\log \left(\left|w_{0} r_{1}^{2}\right|\right)} .
\end{aligned}
$$

As already argued, the size of the $\left|w_{0} r_{1}^{2}\right|,\left|w_{i} r_{j}^{2}+2 H U_{i}\right|$ (for $j=$ $i, i+1)$ terms is large, and so all the logarithms in this expression are positive. Rearranging, we obtain the bound

$$
\begin{aligned}
\log \left(\frac{V_{0}}{V_{1}}\right)+\frac{\log \left(\left|w_{1} r_{1}^{2}+2 H U_{1}\right|\right)}{\log \left(\left|w_{1} r_{2}^{2}+2 H U_{1}\right|\right)} \log \left(\frac{V_{1}}{V_{2}}\right) \\
\quad<-\frac{1}{2} \log \left(\left|w_{0} r_{1}^{2}\right|\right)+\log \left(r_{2} \mathcal{V}\left(r_{2}\right)\right) \frac{\log \left(\left|w_{1} r_{1}^{2}+2 H U_{1}\right|\right)}{\log \left(\left|w_{1} r_{2}^{2}+2 H U_{1}\right|\right)} .
\end{aligned}
$$

Similarly in the five-region case, combining the first five boundary and matching conditions (65)-(69) gives

$$
\begin{gathered}
-\frac{1}{2}>a \log \left(\frac{V_{0}}{V_{1}}\right)+a b \log \left(\frac{V_{1}}{V_{2}}\right)+a b c \log \left(\frac{V_{2}}{V_{3}}\right) \\
+a b c d\left(\log \left(\frac{V_{3}}{V_{4}}\right)-\log \left(r_{4} \mathcal{V}\left(r_{4}\right)\right)\right),
\end{gathered}
$$

where

$$
\begin{gathered}
a=\frac{1}{\log \left(\left|w_{0} r_{1}^{2}\right|\right)}, \quad b=\frac{\log \left(\left|w_{1} r_{1}^{2}+2 H U_{1}\right|\right)}{\log \left(\left|w_{1} r_{2}^{2}+2 H U_{1}\right|\right)}, \\
c=\frac{\log \left(\left|w_{2} r_{2}^{2}+2 H U_{2}\right|\right)}{\log \left(\left|w_{2} r_{3}^{2}+2 H U_{2}\right|\right)}, \quad d=\frac{\log \left(\left|w_{3} r_{3}^{2}+2 H U_{3}\right|\right)}{\log \left(\left|w_{3} r_{4}^{2}+2 H U_{3}\right|\right)},
\end{gathered}
$$

are all positive constants. Rearranging, since by the final boundary condition (70) $\frac{\lambda r_{4}^{2}}{2 U_{4}}>0$, this gives the negative upper bound

$$
\begin{aligned}
& a \log \left(\frac{V_{0}}{V_{1}}\right)+a b \log \left(\frac{V_{1}}{V_{2}}\right)+a b c \log \left(\frac{V_{2}}{V_{3}}\right)+a b c d \log \left(\frac{V_{3}}{V_{4}}\right) \\
&<-\frac{1}{2}+a b c d \log \left(r_{4} \mathcal{V}\left(r_{4}\right)\right) .
\end{aligned}
$$

In order that all four (or six in the five region case) boundary and matching conditions hold, however $V_{i}$ are chosen, they must satisfy the bound (76) (or (78)) for the steady state data used.

Let us finally remark that the $V_{i}$ cannot all be equal. We note that if $V_{i}$ are all equal, then $\frac{V_{i}}{V_{i+1}}=1$ and so $\log \left(\frac{V_{i}}{V_{i+1}}\right)=0$ for each $i$. But then the left side of the bound (76) (or (78), respectively) is zero, which cannot be less than a negative number and so the bound does not hold and the consistency condition is not satisfied.

\section{Application of the stability results}

We now apply the general stability results to specific cases, using realistic data from the literature to select parameter values.

\subsection{Stability of the three region steady state}

Define the quantity

$$
\alpha:=\log \left(\frac{\left|w_{0} r_{1}^{2}\right|}{\left|w_{1} r_{1}^{2}+2 H U_{1}\right|}\right) .
$$

Since $\lambda$ has the same sign as the numerator of (61), if $\alpha>0$, then $\lambda>0$ if $\mu>-\frac{1}{\alpha H} \log \left(\frac{V_{1}}{V_{0}}\right)$, and vice-versa. Alternatively, if $\alpha<0$, then the opposite sign relation holds between the two inequalities.

We assume that $V_{0}<V_{1}$ and $\operatorname{so} \log \left(\frac{V_{1}}{V_{0}}\right)>0$. This is justified physically since the tangential velocity is by definition greater in the eyewall than in the eye, and so it makes sense that the perturbation to the tangential velocity is also greater in the eyewall than in the eye. Also, since $\log \left(\frac{V_{0}}{V_{1}}\right)<0$, we would not have to choose too extreme a value of $V_{2}$ in order that the consistency condition (76) holds.

Since $\log \left(\frac{V_{1}}{V_{0}}\right)>0$ in this case, note that $-\frac{1}{\alpha H} \log \left(\frac{V_{1}}{V_{0}}\right)<0$, when $\alpha>0$ (and $-\frac{1}{\alpha H} \log \left(\frac{V_{1}}{V_{0}}\right)>0$, when $\alpha<0$ ) and so the perturbation is only stable for sufficiently large magnitudes of $\mu$, and when

$$
\operatorname{sgn}(\mu)=-\operatorname{sgn}(\alpha)
$$

In either of the cases $\alpha$ positive or negative, if the perturbation varies sufficiently slowly in the $z$-direction, so that $\mu$ is sufficiently small in magnitude, we see that the perturbation is unstable. 
Stability is only obtained for perturbations with a larger variation in the $z$-direction, and only when the sign of $\mu$ opposes that of $\alpha$. In particular, in either case the perturbation is unstable if it is independent of $z$, ie: when $\mu=0$.

As in section 4.2,

$$
\operatorname{sgn}(\lambda)=\operatorname{sgn}\left(\frac{1}{H} \log \left(\frac{V_{1}}{V_{0}}\right)+\mu \log \left(\frac{\left|\hat{u}_{0}\left(r_{1}\right)\right|}{\left|\hat{u}_{1}\left(r_{1}\right)\right|}\right)\right)
$$

and so the curve separating the stable and unstable parameter regions in the $\left(\mu, \frac{V_{1}}{V_{0}}\right)$ plane (i.e., where $\left.\lambda=0\right)$ is given by

$$
\frac{V_{1}}{V_{0}}=\left(\frac{\left|\hat{u}_{0}\left(r_{1}\right)\right|}{\left|\hat{u}_{1}\left(r_{1}\right)\right|}\right)^{-\mu H}
$$

Following Schubert et al. (2007) and Rozoff et al. (2008), $H=$ $8.79 \times 10^{3} \mathrm{~m}$. The only remaining data needed is $\frac{\left|\hat{u}_{0}\left(r_{1}\right)\right|}{\left|\hat{u}_{1}\left(r_{1}\right)\right|}$. Data for $z$-independent radial velocity is scarce in the literature, however Figures A and B of Willoughby (1979) (graphs of observed radial velocity data) seem to suggest that variations in the radial velocity don't really depend on radial position: there appears to be little pattern to the data for the eye, eyewall and surrounding regions. We therefore choose, for the purpose of an example, to take $\hat{u}$ so that the average flow in each region is the same, i.e. we set

$$
\frac{1}{r_{1}} \int_{0}^{r_{1}} \hat{u}_{0}(r) r d r=\frac{1}{r_{2}-r_{1}} \int_{r_{1}}^{r_{2}} \hat{u}_{1}(r) r d r .
$$

Using the known form of $\hat{u}$, that $\hat{u}_{i}=\frac{U_{i}}{r}+\frac{w_{i} r}{2 H}$, and the fact that $U_{0}=0$, we obtain an expression for $U_{1}$,

$$
U_{1}=\frac{1}{6 H}\left(w_{0} r_{1}^{2}-w_{1}\left(r_{2}^{2}+r_{2} r_{1}+r_{1}^{2}\right)\right) .
$$

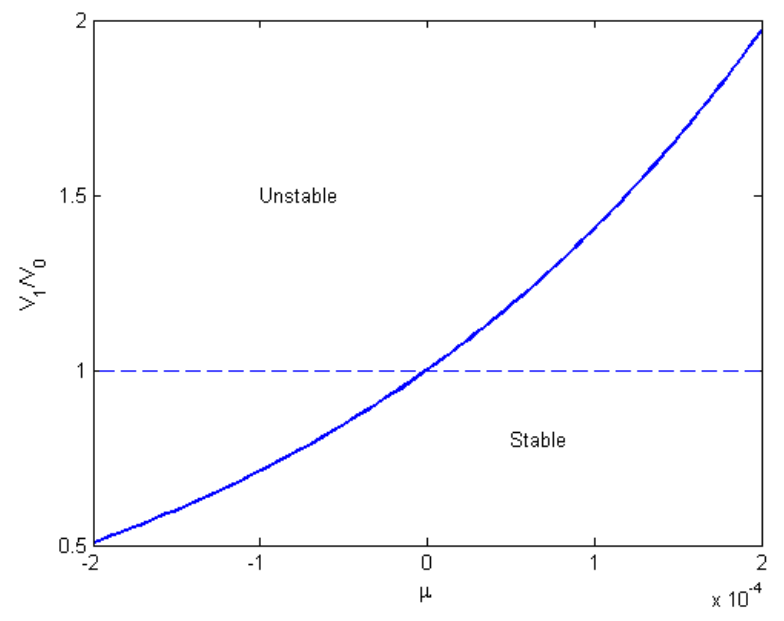

Figure 3. Bifurcation diagram in the $\left(\mu, \frac{V_{1}}{V_{0}}\right)$ plane, with $\frac{\left|\hat{u}_{0}\left(r_{1}\right)\right|}{\left|\hat{u}_{1}\left(r_{1}\right)\right|}=0.68$, and so $\alpha<0$. The solid line is $\lambda=0$, with stability below and instability above this curve. The dashed line shows $V_{0}=V_{1}$. As argued above, we expect $V_{1}>V_{0}$ (above this dashed line) to be the more physically relevant region. These results suggest that for stronger vertical wind in the annular eyewall than in the eye or the outer region, the steady solution will be unstable against modes which decay with height $(\mu<0)$, yet can be stabilized by modes which increase in magnitude with height $(\mu>0)$ in some cases - depending on the relative size of the ratio of tangential wind perturbations and vertical wind profiles.

Now we use data from Schubert et al. (2007), specifically using option A in Figure 5b, taking $r_{1}=10 \mathrm{~km}, r_{2}=20 \mathrm{~km}$, and the approximate values $w_{0}=-1.2 \mathrm{~m} s^{-1}, w_{1}=3 \mathrm{~m} s^{-1}$ from the graph. These values give $U_{1}=-4.2 \times 10^{4}$, and hence we obtain $\frac{\left|\hat{u}_{0}\left(r_{1}\right)\right|}{\left|\hat{u}_{1}\left(r_{1}\right)\right|}=0.68$. With this data, the bifurcation diagram obtained for the neutral stability curve is given in Figure 3. The plot shows the diagram for very small values of $\mu$, since $H \sim$ $O\left(10^{3}\right)-O\left(10^{4}\right)$. Clearly, since the curve is so steep, for larger magnitude $\mu$ (so long as the ratio $\frac{V_{1}}{V_{0}}$ is neither extremely large nor small), we have $\operatorname{sgn}(\lambda)=\operatorname{sgn}(\alpha \mu)$. Recall the $z$-dependence of the perturbation is a factor of $e^{\mu z}$. When $\mu$ is larger than around $10^{-2}$ say, $\mu H \sim O\left(10^{-2} \times 10^{3}\right)=O(10)$ and so the curve in the diagram is very steep. For such values of $\mu$, and for non-extreme (large or small) values of $\frac{V_{1}}{V_{0}}$, it seems the sign of $\mu \alpha$ is the crucial quantity for determining the stability of the steady solution under such a perturbation.

Alternatively, in a situation where $\frac{\left|\hat{u}_{0}\left(r_{1}\right)\right|}{\left|\hat{u}_{1}\left(r_{1}\right)\right|}>1$ (or, $\left.\alpha>0\right)$ the bifurcation diagram will have different shape. For example, if we take $\frac{\left|\hat{u}_{0}\left(r_{1}\right)\right|}{\left|\hat{u}_{1}\left(r_{1}\right)\right|}=1.5$, then the bifurcation diagram is as given in Figure 4.

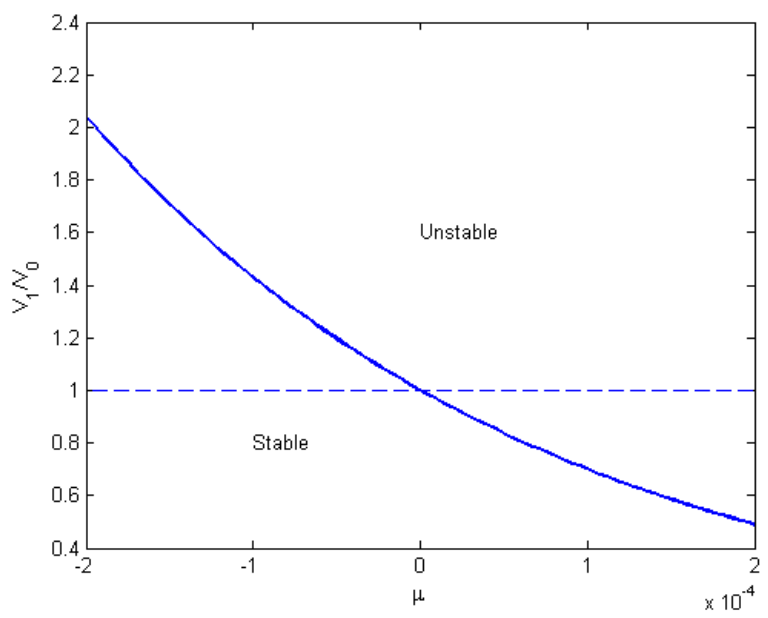

Figure 4. Bifurcation diagram in the $\left(\mu, \frac{V_{1}}{V_{0}}\right)$ plane, with $\alpha>0$. As in Figure 3, the solid line is $\lambda=0$, with stability below and instability above this curve, and the dashed line shows $V_{0}=V_{1}$. These results suggest that for stronger vertical wind in eye, the steady solution will be unstable against modes which decay with height $(\mu>0)$, yet can be stabilized by modes which decrease in magnitude with height $(\mu<0)$ in some cases - depending again on the relative size of the ratio of tangential wind perturbations and vertical wind profiles.

These results suggest a change in the stability or instability of the steady state tropical cyclone depending on which of the subsidence within the eye or the rising air within the eyewall is dominant, and that perturbations which increase in tangential velocity with height may be stabilizing or destabilizing, depending on other parameters. In the case where perturbations are uniform in height, we always find an instability for $V_{1}>V_{0}$, i.e. when the perturbed tangential velocity is greater in the eyewall than in the eye. Therefore, a tangential wind velocity profile which varies in $z$ appears necessary for stability of steady state vortex solutions under the balanced vortex model we consider.

\subsection{Stability of the five region steady state}

The five-region model is slightly more complicated than the three region case. The choice of $V_{i}$ is constrained not only by the consistency condition (77), but also by the fact that all three expressions for $\lambda$ given by all three of (71)-(73) must be the same. Essentially, by equating these three equations we eliminate two of the ratios, e.g. we can consider $\frac{V_{2}}{V_{1}}$ and $\frac{V_{3}}{V_{2}}$ to be given in terms of 
$\frac{V_{1}}{V_{0}}$, and then the consistency condition may be written

$$
\begin{aligned}
& \log \left(\frac{V_{0}}{V_{1}}\right)\left(a-\frac{b}{A}(B+c C)\right)+a b c d \log \left(\frac{V_{3}}{V_{4}}\right) \\
< & -\frac{1}{2}-a b c d \frac{\lambda r_{4}^{2}}{2 U_{4}}+b \mu H\left(\frac{1}{A} \log \left(\frac{\left|w_{0} r_{1}^{2}\right|}{\left|w_{1} r_{1}^{2}+2 H U_{1}\right|}\right)(B+c C)\right. \\
& \left.-\log \left(\frac{\left|w_{1} r_{2}^{2}+2 H U_{1}\right|}{\left|w_{2} r_{2}^{2}+2 H U_{2}\right|}\right)-c \log \left(\frac{\left|w_{2} r_{3}^{2}+2 H U_{2}\right|}{\left|w_{3} r_{3}^{2}+2 H U_{3}\right|}\right)\right)
\end{aligned}
$$

where the constants $a, b, c, d>0$ are as defined in the previous subsection, while

$$
\begin{gathered}
A=\log \left(\left|w_{1} r_{1}^{2}+2 H U_{1}\right|^{1 / w_{1}}\left|w_{0} r_{1}^{2}\right|^{-1 / w_{0}}\right), \\
B=\log \left(\left|w_{2} r_{2}^{2}+2 H U_{2}\right|^{1 / w_{2}}\left|w_{1} r_{2}^{2}+2 H U_{1}\right|^{-1 / w_{1}}\right), \\
C=\log \left(\left|w_{3} r_{3}^{2}+2 H U_{3}\right|^{1 / w_{3}}\left|w_{2} r_{3}^{2}+2 H U_{2}\right|^{-1 / w_{2}}\right) .
\end{gathered}
$$

As argued in section 4.3, these constants satisfy $A, C>0$ and $B<$ 0. If $\log \left(\frac{V_{0}}{V_{1}}\right)$ is chosen, then (84) gives a bound on $\log \left(\frac{V_{3}}{V_{4}}\right)$, which must be satisfied if the perturbation $V$ is to satisfy all six boundary conditions.

Since we have now imposed that our three expressions for $\lambda$ (71)-(73) are the same, we can find the sign of $\lambda$ from any one of them. If, as above, we choose to specify $\frac{V_{1}}{V_{0}}$ and then consider $\frac{V_{2}}{V_{1}}$ and $\frac{V_{3}}{V_{2}}$ to be functions of $\frac{V_{1}}{V_{0}}$, it makes sense to look at equation (71) for the sign of $\lambda$. Note this is identical to the condition in the three region case, and so in fact we obtain the same result: if $\alpha \gtrless 0$, then $\lambda>0$ if $\mu \gtrless-\frac{1}{\alpha H} \log \left(\frac{V_{1}}{V_{0}}\right)$, and vice-versa.

If we choose $V_{0}$ and $V_{1}$ such that $V_{0}<V_{1}$, then the ratios $\frac{V_{2}}{V_{1}}$ and $\frac{V_{3}}{V_{2}}$ are specified in terms of $\frac{V_{1}}{V_{0}}$. Bounds on these ratios may be ascertained from the equivalence of (71)-(73) by noting that, since $V_{0}<V_{1}$ :

$$
\begin{aligned}
0<\frac{A}{B} & \left(\log \left(\frac{V_{2}}{V_{1}}\right)+\mu H \log \left(\frac{\left|w_{1} r_{2}^{2}+2 H U_{1}\right|}{\left|w_{2} r_{2}^{2}+2 H U_{2}\right|}\right)\right) \\
& -\mu H \log \left(\frac{\left|w_{0} r_{1}^{2}\right|}{\left|w_{1} r_{1}^{2}+2 H U_{1}\right|}\right),
\end{aligned}
$$

and

$$
\begin{aligned}
0<\frac{A}{C} & \left(\log \left(\frac{V_{3}}{V_{2}}\right)+\mu H \log \left(\frac{\left|w_{2} r_{3}^{2}+2 H U_{2}\right|}{\left|w_{3} r_{3}^{2}+2 H U_{3}\right|}\right)\right) \\
& -\mu H \log \left(\frac{\left|w_{0} r_{1}^{2}\right|}{\left|w_{1} r_{1}^{2}+2 H U_{1}\right|}\right) .
\end{aligned}
$$

To determine the sign of $\lambda$, we note that, since $\log \left(\frac{V_{1}}{V_{0}}\right)>0$, the perturbation is only stable for sufficiently large magnitudes of $\mu$, when $\mu$ has opposite sign to $\alpha$.

For a simple example, we examine in more detail the $\mu=0$ case. If the perturbation is $z$-independent, so $\mu=0$, then the equations equating the expressions (71)-(73) for $\lambda$ are much simpler. The values of $\frac{V_{2}}{V_{1}}$ and $\frac{V_{3}}{V_{2}}$ are given by:

$$
\begin{aligned}
& \log \left(\frac{V_{2}}{V_{1}}\right)=\frac{B}{A} \log \left(\frac{V_{1}}{V_{0}}\right) \\
& =\frac{\log \left(\left|w_{2} r_{2}^{2}+2 H U_{2}\right|^{1 / w_{2}}\left|w_{1} r_{2}^{2}+2 H U_{1}\right|^{-1 / w_{1}}\right)}{\log \left(\left|w_{1} r_{1}^{2}+2 H U_{1}\right|^{1 / w_{1}}\left|w_{0} r_{1}^{2}\right|^{-1 / w_{0}}\right)} \log \left(\frac{V_{1}}{V_{0}}\right),
\end{aligned}
$$

and

$$
\begin{aligned}
& \log \left(\frac{V_{3}}{V_{2}}\right)=\frac{C}{A} \log \left(\frac{V_{1}}{V_{0}}\right) \\
& =\frac{\log \left(\left|w_{3} r_{3}^{2}+2 H U_{3}\right|^{1 / w_{3}}\left|w_{2} r_{3}^{2}+2 H U_{2}\right|^{-1 / w_{2}}\right)}{\log \left(\left|w_{1} r_{1}^{2}+2 H U_{1}\right|^{1 / w_{1}}\left|w_{0} r_{1}^{2}\right|^{-1 / w_{0}}\right)} \log \left(\frac{V_{1}}{V_{0}}\right) .
\end{aligned}
$$

Since $A, C>0$ and $B<0$, and we have chosen $V_{0}<V_{1}$, these relations imply $V_{2}<V_{1}$ and $V_{3}>V_{2}$. Requiring the tangential perturbation to be greater in the inner eyewall than the eye, $V_{0}<$ $V_{1}$, which is arguably the most physically relevant case since the greater perturbation is in the region where there is a higher leading order value, imposes that the perturbations in the other pairs of neighbouring regions follow the same pattern: the perturbation is greater in the region in which we expect the greater leading order velocity. For instance, $V_{1}>V_{2}$ and we expect higher leading order tangential wind in the inner eyewall than the moat, and $V_{2}<V_{3}$, and we expect lower leading order tangential wind in the moat than in the outer eyewall.

The consistency condition gives

$$
\begin{aligned}
\log \left(\frac{V_{0}}{V_{1}}\right)\left(a-\frac{b}{A}(B+c C)\right) & +a b c d \log \left(\frac{V_{3}}{V_{4}}\right) \\
& <-\frac{1}{2}+a b c d \log \left(r_{4} \mathcal{V}\left(r_{4}\right)\right)
\end{aligned}
$$

which, since $a, b, c, d>0$, gives an upper bound for $\log \left(\frac{V_{3}}{V_{4}}\right)$. Regardless of the sign of this upper bound, it is clearly possible to choose $\log \left(\frac{V_{3}}{V_{4}}\right)<0$, and so have the far-field perturbation smaller than the perturbation in the outer eyewall, continuing the pattern of greater perturbations in regions of greater leading order tangential velocity.

Although the calculations are considerably messier in the general $z$-dependent $(\mu \neq 0)$ case, a similar process can be undertaken to obtain the values of the ratios $\frac{V_{2}}{V_{1}}$ and $\frac{V_{3}}{V_{2}}$ (in terms of $\mu$ and $\frac{V_{0}}{V_{1}}$ ) and a bound on $\frac{V_{4}}{V_{3}}$ via the consistency condition (84). As above, the sign of $\lambda$ is then given by any one of (71)(73), and in the $\mu=0$ case we obtain $\lambda>0$ and so an unstable perturbation. As seen through this analysis of the five region model, the expression for $\lambda$ may be taken as precisely that given in (61), and so the bifurcation diagrams Figures 3 and 4 also hold for the five region model with this data for the eye and inner eyewall.

\section{Discussion}

We have analytically investigated the linear stability of steady state vortex solutions to the transverse circulation equations under general pertubations to the structure of the vortex. To match more realistic tropical cyclone structures, we considered three region cases (eye, eyewall, far-field) and five region cases (eye, inner eyewall, moat, outer eyewall, far-field) in order to approximate stationary state tropical cyclones with single or double (concentric) eyewalls. In order to make progress with the analysis, the piecewise steady state solutions we considered were axisymmetric, barotropic, and largely independent of $z$ (except where $z$ is suspected to play a large role). While somewhat idealized, we verify that such solutions are still solutions of the transverse circulation equations, while other steady states considered in the literature fail one of the necessary criteria, and are not truly solutions of the steady state problem. (That is to say, previous solutions did not exactly satisfy one or more of the transverse circulation equations at steady state). Therefore, while one can view such solutions in the literature as "approximate solutions", one should not use them when performing a stability analysis, as the stability analysis will be rather sensitive to the form of the base state solutions to the stationary problem. This was the primary motivation in our obtaining general solutions to the transverse circulation equations from scratch.

The general stability analysis involves defining small structural perturbations to the steady state vortex solutions. These perturbations must still satisfy the linear eigenvalue problem defined by the linearised transverse circulation equations (which are linearised about a particular steady state vortex solution). 
Solving the relevant eigenvalue problem, we are able to obtain the relevant spectral parameter which determines if a given perturbation will decay in time (giving stable steady states) or grow in time (meaning the corresponding steady state solution is unstable). The stability of a given steady state solution was shown to be determined by the sign of an expression involving the ratio of steady radial velocity $\hat{u}_{i}\left(r_{j}\right)$ across the boundaries between regions, on the $z$-dependence of the perturbation $\mu$, and on the relative sizes of the magnitudes of the perturbation $\frac{V_{i}}{V_{i+1}}$. Other parameters factor into the stability criteria, as well, but since they can be estimated experimentally or via observation, they are not truly "free" parameters but instead will come from the particular atmospheric conditions observed at a given time. We found that for $z$-independent perturbations, both the three and five region cases are unstable when considering physically relevant parameter values for both the steady state solution and the perturbation sizes. Note that the perturbation parameters were required to satisfy a set of consistency conditions (mandated by matching the different regions of the eigenvalue problem to obtain a continuous solution profile for the perturbations, which reduces the number of degrees of freedom when choosing from arbitrary perturbations), and the choice of certain values of these parameters was discussed.

Note that while our analysis can be used for tropical cyclones with single or double eyewalls, it says nothing about the transition between the two. While a stable vortex structure will maintain its form over time, is is not possible to know how an unstable structure will evolve over time without solving the fully nonlinear transverse circulation equations. It is the transient dynamics that will dictate, for instance, whether a single eyewall steady state transitions into a concentric eyewall structure. Therefore, one should view the stability analysis as a binary test of whether or not a particular state is likely to exist. Note also that many parameter values are not time static in practice, but will change in time. Therefore, while the stability test gives similar stability/instability regions for both single and double eyewall steady state tropical cyclones, it is likely that the double eyewall will exist, in practice, for parameter values which correspond to instability. In contrast, we suspect that the most stable steady states for the single eyewall steady states would correspond to parameter regimes found in annular tropical cyclones (Knaff et al. 2003), as the annular state is known to be robust in time. One benefit of the analytical approach we take is that all of the stability results are given explicitly for arbitrary parameters (rather than numerical simulations for one set of parameters), and hence one can evaluate our results at any particular parameter values of interest, in order to determine stability of a particular solution corresponding to such parameter values.

Clearly it would be beneficial to be able to consider more general models, retaining $z$-dependence in the steady state solution and not requiring the barotropic assumption on $v$ at lowest order. However, as seen from our analysis, such complications may yield very complicated equations even at steady state, which may not be amenable to analytical techniques. For such cases, one would likely need to resort to numerical methods. As discussed in Section 1, numerical approaches have been employed in the past in order to better understand the dynamics of tropical cyclone eyewalls (see Schubert et al. (1999), Nolan and Montgomery (2002), Naylor and Schecter (2014)). So it is likely that the analytical approach has been pushed about as far as is possible. Still, the analytical stability test has given us a crude but qualitatively reasonable understanding of parameter regimes for which a steady state tropical cyclone may be stable or unstable under structural perturbations.

It is important to note that there will be an impact of boundary layer and convective processes on the stability results presented here. Wu et al. (2016), for example, show the enormous impact of diabatic heating in stabilizing TC ring structures, thereby making them robust against instabilities. Planetary boundary layer forcing can also have a large impact in driving TC configurations. Therefore, instability in the model we consider may indeed be stabilized due to boundary layer and convective processes. As such, results stability results may have limited applicability to realistic TCs and ERCs since the governing dynamics are strongly impacted by latent heating and nonlinear boundary layer forcing, which are neglected in the model we consider. For instance, this suggests that the mechanisms we study are similar for both the single and concentric eyewall configurations, resulting in similar linear stability results, and that boundary layer and convective influences likely lead to more discernible differences between the stability of the two distinct configurations when such terms are included. Still, the linear stability analysis is useful in that it allows us to isolate dynamics that may nonetheless impact realistic TCs and ERCs. Future work could be to consider a more complicated model which accounts for boundary layer and convective processes, and then to derive analogous stability results making use of our approach. In particular, the approach we use here is potentially useful provided that steady state solutions to such a more complicated model exist.

\section{Acknowledgement}

This publication is based on work partially supported by the EPSRC Centre For Doctoral Training in Industrially Focused Mathematical Modelling (EP/L015803/1), by Exeter College, University of Oxford, and by the Mathematical Institute, University of Oxford.

\section{References}

Abarca SF, Montgomery MT. 2014. Departures from axisymmetric balance dynamics during secondary eyewall formation. Journal of the Atmospheric Sciences 71(10): 3723-3738.

Dritschel D, Waugh D. 1992. Quantification of the inelastic interaction of unequal vortices in two-dimensional vortex dynamics. Physics of Fluids A: Fluid Dynamics (1989-1993) 4(8): 1737-1744.

Emanuel KA. 1987. An air-sea model of intraseasonal oscillations in the tropics. Journal of the atmospheric sciences 44(16): 2324-2340.

Huang YH, Montgomery MT, Wu CC. 2012. Concentric eyewall formation in typhoon sinlaku (2008). part ii: Axisymmetric dynamical processes. Journal of the Atmospheric Sciences 69(2): 662-674.

Knaff JA, Kossin JP, DeMaria M. 2003. Annular hurricanes. Weather and Forecasting 18(2): 204-223.

Kossin JP. 2015. Hurricane wind-pressure relationship and eyewall replacement cycles. Weather and Forecasting 30(1): 177-181.

Kossin JP, Eastin MD. 2001. Two distinct regimes in the kinematic and thermodynamic structure of the hurricane eye and eyewall. Journal of the atmospheric sciences 58(9): 1079-1090.

Kossin JP, McNoldy BD, Schubert WH. 2002. Vortical swirls in hurricane eye clouds. Monthly Weather Review 130(12): 3144-3149.

Kossin JP, Schubert WH. 2001. Mesovortices, polygonal flow patterns, and rapid pressure falls in hurricane-like vortices. Journal of the atmospheric sciences 58(15): 2196-2209.

Kossin JP, Schubert WH. 2003. Diffusion versus advective rearrangement of a circular vortex sheet. Journal of the atmospheric sciences 60(3): 586-589.

Kossin JP, Schubert WH. 2004. Mesovortices in hurricane isabel. Bulletin of the American Meteorological Society 85(2): 151-153.

Kossin JP, Schubert WH, Montgomery MT. 2000. Unstable interactions between a hurricanes primary eyewall and a secondary ring of enhanced vorticity. Journal of the atmospheric sciences 57(24): 3893-3917.

Kuo H, Lin L, Chang C, Williams R. 2004. The formation of concentric vorticity structures in typhoons. Journal of the atmospheric sciences 61(22): 2722-2734.

Kuo HC, Schubert WH, Tsai CL, Kuo YF. 2008. Vortex interactions and barotropic aspects of concentric eyewall formation. Monthly Weather Review 136(12): 5183-5198.

Montgomery MT, Kallenbach RJ. 1997. A theory for vortex rossby-waves and its application to spiral bands and intensity changes in hurricanes. Quarterly Journal of the Royal Meteorological Society 123(538): 435-465. 
Naylor J, Schecter DA. 2014. Evaluation of the impact of moist convection on the development of asymmetric inner core instabilities in simulated tropical cyclones. Journal of Advances in Modeling Earth Systems 6(4): 1027-1048.

Nolan DS, Montgomery MT. 2002. Nonhydrostatic, three-dimensional perturbations to balanced, hurricane-like vortices. part i: Linearized formulation, stabilty, and evolution. Journal of the atmospheric sciences 59(21): 2989-3020.

Nong S, Emanuel K. 2003. A numerical study of the genesis of concentric eyewalls in hurricanes. Quarterly Journal of the Royal Meteorological Society 129(595): 3323-3338.

Rozoff CM, Kossin JP, Schubert WH, Mulero PJ. 2009. Internal control of hurricane intensity variability: The dual nature of potential vorticity mixing. Journal of the Atmospheric Sciences 66(1): 133-147.

Rozoff CM, Nolan DS, Kossin JP, Zhang F, Fang J. 2012. The roles of an expanding wind field and inertial stability in tropical cyclone secondary eyewall formation. Journal of the Atmospheric Sciences 69(9): 2621-2643.

Rozoff CM, Schubert WH, Kossin JP. 2008. Some dynamical aspects of tropical cyclone concentric eyewalls. Quarterly Journal of the Royal Meteorological Society 134(632): 583-593.

Rozoff CM, Schubert WH, McNoldy BD, Kossin JP. 2006. Rapid filamentation zones in intense tropical cyclones. Journal of the atmospheric sciences 63(1): 325-340.

Schubert WH, Montgomery MT, Taft RK, Guinn TA, Fulton SR, Kossin JP, Edwards JP. 1999. Polygonal eyewalls, asymmetric eye contraction, and potential vorticity mixing in hurricanes. Journal of the atmospheric sciences 56(9): 1197-1223.

Schubert WH, Rozoff CM, Vigh JL, McNoldy BD, Kossin JP. 2007. On the distribution of subsidence in the hurricane eye. Quarterly Journal of the Royal Meteorological Society 133(624): 595-605.

Shapiro LJ, Willoughby HE. 1982. The response of balanced hurricanes to local sources of heat and momentum. Journal of the Atmospheric Sciences 39(2): 378-394.

Sun YQ, Jiang Y, Tan B, Zhang F. 2013. The governing dynamics of the secondary eyewall formation of typhoon sinlaku (2008). Journal of the Atmospheric Sciences 70(12): 3818-3837.

Terwey WD, Montgomery MT. 2008. Secondary eyewall formation in two idealized, full-physics modeled hurricanes. Journal of Geophysical Research: Atmospheres 113(D12).

Wang H, Wu CC, Wang Y. 2016. Secondary eyewall formation in an idealized tropical cyclone simulation: Balanced and unbalanced dynamics. Journal of the Atmospheric Sciences 73(10): 3911-3930.

Williams GJ, Taft RK, McNoldy BD, Schubert WH. 2013. Shock-like structures in the tropical cyclone boundary layer. Journal of Advances in Modeling Earth Systems 5(2): 338-353.

Williams Jr GJ. 2016. The inner core thermodynamics of the tropical cyclone boundary layer. Meteorology and Atmospheric Physics : 1-20.

Willoughby H. 1979. Forced secondary circulations in hurricanes. Journal of Geophysical Research: Oceans 84(C6): 3173-3183.

Willoughby H, Clos J, Shoreibah M. 1982. Concentric eye walls, secondary wind maxima, and the evolution of the hurricane vortex. Journal of the Atmospheric Sciences 39(2): 395-411.

Wu CC, Wu SN, Wei HH, Abarca SF. 2016. The role of convective heating in tropical cyclone eyewall ring evolution. Journal of the Atmospheric Sciences 73(1): 319-330.

Yang YT, Kuo HC, Hendricks EA, Peng MS. 2013. Structural and intensity changes of concentric eyewall typhoons in the western north pacific basin. Monthly Weather Review 141(8): 2632-2648.

Zhang F, Tao D, Sun YQ, Kepert JD. 2017. Dynamics and predictability of secondary eyewall formation in sheared tropical cyclones. Journal of Advances in Modeling Earth Systems 9(1): 89-112.

Zhou X, Wang B. 2011. Mechanism of concentric eyewall replacement cycles and associated intensity change*. Journal of the Atmospheric Sciences 68(5): 972-988. 